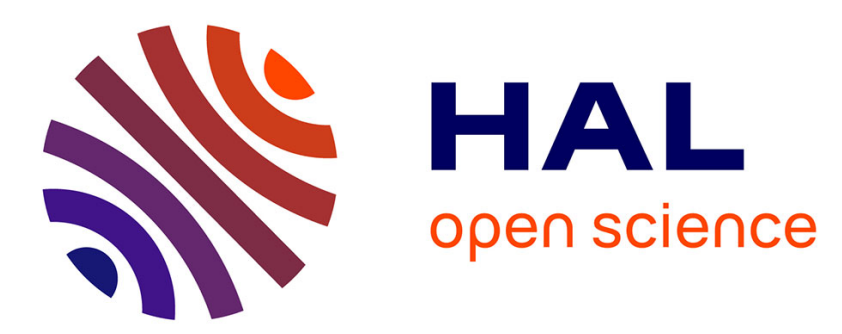

\title{
Transmission iatrogène des protéines $\beta$-amyloïde et tau : importance clinique et pour la recherche biomédicale sur la maladie d'Alzheimer
}

Marc Dhenain

\section{- To cite this version: \\ Marc Dhenain. Transmission iatrogène des protéines $\beta$-amyloïde et tau : importance clinique et pour la recherche biomédicale sur la maladie d'Alzheimer. Bulletin de l'Académie Nationale de Médecine, 2021, 205 (7), pp.792-799. 10.1016/j.banm.2021.04.018 . hal-03447469}

\author{
HAL Id: hal-03447469 \\ https://hal.science/hal-03447469
}

Submitted on 24 Nov 2021

HAL is a multi-disciplinary open access archive for the deposit and dissemination of scientific research documents, whether they are published or not. The documents may come from teaching and research institutions in France or abroad, or from public or private research centers.
L'archive ouverte pluridisciplinaire HAL, est destinée au dépôt et à la diffusion de documents scientifiques de niveau recherche, publiés ou non, émanant des établissements d'enseignement et de recherche français ou étrangers, des laboratoires publics ou privés. 
Transmission iatrogène des protéines $\beta$-amylö̈de et tau : Importance clinique et pour la recherche biomédicale sur la maladie d'Alzheimer

Iatrogenic transmission of $\beta$-amyloid and tau proteins: Importance for clinical and biomedical research on Alzheimer's disease

\title{
Marc DHENAIN
}

Laboratoire des Maladies Neurodégénératives

UMR9199 CEA, CNRS, Université Paris Saclay - MIRCen

18 Route du Panorama, 92265 Fontenay aux Roses. France

* Auteur correspondant : Laboratoire des Maladies Neurodégénératives, UMR9199 CEA, CNRS, UPSaclay, UPSud - MIRCen - 18 Route du Panorama, 92265 Fontenay aux Roses.

\begin{abstract}
E-mail : Marc.Dhenain@cea.fr
\end{abstract}
https://orcid.org/0000-0001-8804-4101

Tirés à part : Docteur Marc DHENAIN, même adresse 


\section{RÉSUMÉ}

Depuis la découverte du caractère transmissible des maladies à prions, d'autres maladies neurodégénératives ont été soupçonnées d'être transmissibles. La maladie d'Alzheimer est caractérisée par des dépôts cérébraux de peptide $\beta$-amylö̈de (Aß, sous la forme de plaques et angiopathie amyloüdes) et de protéines Tau hyperphosphorylées (dégénérescences neurofibrillaires, fibres tortueuses, couronnes de prolongements cellulaires dilatés autour des plaques amylö̈des neuritiques). Plusieurs études épidémiologiques suggèrent 75 cas de transmissions iatrogènes des dépôts $\beta$-amylö̈des chez l'Homme suite à différentes procédures médico-chirurgicales. Cette transmission aurait dans certains cas un impact clinique majeur pouvant entrainer la mort par hémorragies cérébrales. Au-delà de cette importance clinique, la transmission des protéines $\beta$-amylö̈de et tau a permis de créer des nouveaux modèles animaux afin d'explorer les mécanismes fondamentaux associés à cette maladie. Ces modèles sont en train de révolutionner la recherche sur les maladies neurodégénératives.

Mots-Cles : AmyloÏDE, Maladie D'Alzheimer, Modeles animaux, TaU, Transmission.

\section{SUMMARY}

52 Since the discovery of the transmissible nature of prion diseases, other neurodegenerative diseases have 53 been suspected of being transmissible. Alzheimer's disease is characterized by brain deposits of $\beta$ 54 amyloid peptide (Aß, forming amyloid plaques and angiopathy) and hyperphosphorylated Tau proteins (neurofibrillary tangles, neuropil thread, neuritic plaques). Several epidemiological studies suggest the iatrogenic transmission of $\beta$-amyloid deposits in 75 humans following various medico-surgical procedures. This transmission would have, in some cases, a major clinical impact that could lead to fatality by cerebral hemorrhages. Beyond this clinical importance, the transmission of $\beta$-amyloid and tau proteins has allowed the creation of novel animal models to explore fundamental mechanisms

60 associated to Alzheimer's disease. These models are revolutionizing research on neurodegenerative 61 diseases. 


\section{TRANSMISSION IATROGENE DES MALADIES A PRION}

Les encéphalopathies spongiformes transmissibles ou maladies à prions sont des maladies neurodégénératives hétérogènes mais toujours rapidement fatales touchant l'Homme ou l'animal. Elles sont causées par l'accumulation intracérébrale d'une protéine prion mal conformée. Différentes formes de maladies à prions humaines ont été décrites, dont la maladie de Creutzfeldt-Jakob, le syndrome de Gerstmann-Sträussler-Scheinker, l'insomnie fatale familiale ou sporadique et le Kuru. Les maladies à prions sont décrites sous trois formes: sporadique, génétique et infectieuse. Les formes sporadiques représentent environ 13000 cas par an dans le monde tandis que les formes génétiques sont plus rares (1000 cas par an). Les formes infectieuses sont appelées encéphalopathies spongiformes transmissibles et peuvent être d'origine alimentaire ou iatrogène. Elles ont été décrites pour la première fois par Gajdusek qui étudiait le Kuru, une maladie neurodégénérative qui touchait des aborigènes de PapouasieNouvelle-Guinée. Ces derniers se contaminaient en consommant le corps des défunts de leur tribu lors de rites anthropophagiques mortuaires. Gajdusek a démontré la transmissibilité de la maladie en inoculant, par voie intracérébrale, le cerveau de personnes touchées à des chimpanzés qui ont développé la pathologie (1). Cette maladie qui a touché environ 2700 personnes a été éradiquée en arrêtant les rites cannibales (2). La transmission alimentaire de maladies à prion a été médiatisée lors de la "crise de la vache folle" dans les années 1990. 231 personnes ayant mangé de la viande bovine ont été contaminées (3). Les transmissions iatrogènes sont, elles, liées à des procédures chirurgicales ou médicales. 470 cas ont été décrits entre 1970 et aujourd'hui (mars 2021) (4) (Table 1). Les procédures chirurgicales les plus contaminantes sont liées à la réalisation de greffes avec des tissus issus de dure-mère (228 cas) et en particulier avec la préparation de dure-mère lyophylisée commercialisée jusqu'à 1992 sous le nom de "Lyodura" par une firme allemande (5). Ce produit était utilisé lors d'actes chirurgicaux entre autre lors de greffes de dure-mère, mais également, dans $20 \%$ des cas lors de procédures orthopédiques, otologiques, dentaires, urologiques, gynécologiques et cardiaques au cours desquelles les extraits de dure-mère servaient à colmater des organes ou à remplacer/réparer diverses parois tissulaires (trachée, plèvre...) (5). Par exemple, il servait à réaliser des embolisations de tumeurs périphériques très vascularisées, afin d'occlure les vaisseaux irriguant les tumeurs (6). D'autres cas très rares de contaminations chirurgicales sont liés à l'utilisation d'instruments ou de sondes d'électro-encéphalogrammes contaminés (6 cas) ou de greffes de cornées ( 2 cas). Des mesures ont été mises en place en France pour réduire le risque de transmission des maladies à prion lors d'actes chirurgicaux (7). Les contaminations 
suite aux procédures médicales concernent principalement des traitements par hormone de croissance (226 cas) ou gonadotrophique (4 cas) extraites de cerveaux de cadavres. Des contaminations suite à l'utilisation de produits dérivés du sang ont aussi été rapportées (culots globulaires, 3 cas (4)). L'utilisation des hormones d'origine humaine est arrêtée depuis 1985 en France. En ce qui concerne les culots globulaires, plusieurs mesures de protections dont la déleucocytation systématique des produits sanguins sont en place depuis 1998.

\section{TRANSMISSION IATROGENE DES LESIONS ASSOCIEES A LA MALADIES D'ALZHEIMER}

La maladie d'Alzheimer est la maladie neurodégénérative la plus répandue actuellement avec un nombre de cas environ 4000 fois plus important que les maladies à prions. Comme les maladies à prion elle présente des formes sporadiques (prévalence d'environ 50 millions dans le monde, ce qui correspond à une incidence approximative de 5 millions de cas par an, si on considère que la maladie évolue pendant 10 ans) et des formes génétiques à transmission autosomale dominante (prévalence de environ un million de cas par an). Cette maladie est caractérisée par une longue phase asymptomatique qui commence au moins 10 à 20 ans avant les premiers signes de la maladie et une phase clinique qui dure également 10 ans (8). Les lésions microscopiques de la maladie d'Alzheimer sont les dépôts extracellulaires de protéines $\mathrm{A} \beta$ mal conformées et les altérations liées à des dépôts intracellulaires de protéines tau anormalement phosphorylées. Les dépôts extracellulaires de protéines $A \beta$ mal conformées peuvent prendre des formes multiples : dépôts diffus, dépôts focaux, plaques neuritiques ou angiopathie amyloïde par exemple (9). Des formes solubles d'oligomères d'A $\beta$ sont également présentes (10). Les lésions contenant des protéines tau anormales sont les dégénérescences neurofibrillaires (accumulations dans le corps cellulaire des neurones), les fibres tortueuses (accumulations dans le neuropile, principalement dans les dendrites) et les couronnes de prolongements cellulaires dilatés autour des plaques amyloïdes neuritiques (9). Les formes solubles des protéines tau anormales ont probablement également un rôle important dans le processus pathologique de la maladie (11). Les lésions contenant des protéines tau sont caractérisées par leur capacité à diffuser de façon stéréotypée dans le cerveau au cours de l'évolution de la maladie $(12,13)$. Cette diffusion permet de définir des stades de la maladie (6 stades de Braak). Au cours des stades I et II, la pathologie atteint le cortex transentorhinal (stade I) puis entorhinal (stade II). Lors des stades III-IV (stades limbiques), elle gagne progressivement l'hippocampe, puis l'isocortex temporal qui reste faiblement touché. Lors des stades V-VI, les autres aires isocorticales sont touchées en commençant par les aires 
associatives frontales ou parieto-occipito-temporales $(12,13)$. Cette progression de la

131 pathologie semble suivre des voies de connexions entre les régions, ce qui pourrait suggérer une propagation des protéines tau le long des voies nerveuses. Les dépôts de protéine $\beta$ amyloïdes sont eux rapidement présents dans l'ensemble du néocortex. Une diffusion se produit

134 lors de l'évolution de ces dépôts and touchant d'abord les zones allocorticales (dont 135 l'hippocampe) puis les régions sous-corticales telles que les noyaux gris centraux, le tronc 136 cérébral et le cervelet (14).

137 Depuis la découverte du caractère transmissible des maladies à prions, la maladie 138 d'Alzheimer a été soupçonnée d'être également transmissible. Cependant, pendant longtemps, 139 les études épidémiologiques ne supportaient pas une étiologie infectieuse de la maladie 140 d'Alzheimer (15-17) et aucune augmentation de l'incidence des formes cliniques de maladie 141 d'Alzheimer n'a été rapportée chez les receveurs d'hormones de croissance issues de cadavres 142 jusqu'à présent (18). Une première étude publiée en 2006 avait pourtant démontré la présence 143 de lésions $\beta$-amyloïdes chez un patient de 28 ans atteint de maladie de Creutzfeldt-Jakob 144 iatrogène après greffe durale (19). En 2015, une deuxième étude a révélé une amyloïdose chez 145 quatre jeunes sujets traités par des hormones de croissance dérivées de cerveaux de cadavres 146 (20). Cet article a relancé une série d'études montrant la transmissibilité de l'amyloïdose 147 cérébrale suite à l'utilisation de cette hormone $(21,22)$ ou suite à des greffes de dure-mère 148 présentant des dépôts amyloïdes $(19,23,24)$. L'interprétation de cette première série d'études 149 était délicate car ces sujets présentaient également une maladie de Creutzfeldt-Jakob iatrogène. 150 L'apparition de ces lésions chez des personnes ayant reçu des greffes de dure-mère et décédées 151 sans Creutzfeldt-Jakob a finalement convaincu d'une possible transmission de lésions $\beta$ 152 amyloïdes (25). Des travaux épidémiologiques plus récents ont révélé que les patients contaminés développent majoritairement une angiopathie amyloïde, sévère, pouvant induire des 154 hémorragies cérébrales mortelles $(26,27)$. Des contaminations par gestes chirurgicaux, pas 155 forcément associés à une greffe de dure-mère, ont finalement été mis en évidence (27). 156 L'ensemble des données épidémiologiques chez l'homme suggèrent donc qu'une contamination 157 entraîne une angiopathie amyloïde associée à des plaques dans le parenchyme cérébral mais 158 n'entraîne pas de tauopathie. Un des points communs entre ces transmissions est le très long 159 délai (souvent plus de 20 ans) entre l'acte potentiellement contaminant et la déclaration de la 160 pathologie. La nature rétrospective des études menées limite la validation formelle d'une 161 relation causale entre les actes médico-chirurgicaux supposés contaminants et l'apparition de la 162 pathologie amyloïde. Cependant, la transmission expérimentale d'amyloïde à des primates par 
163 inoculation d'extraits de cerveaux humains Alzheimer a confirmé la possible transmission

164 d'amyloïde- $\beta(28,29)$. Les primates ont également développé une tauopathie, une atrophie cérébrale et des troubles comportementaux. Chez l'homme, les troubles cognitifs n'ont pas été évalués précisément mais les données actuelles ne suggèrent pas d'atteinte cognitive évoquant la maladie d'Alzheimer. La maladie développée est plutôt une amyloïdose à prédominance vasculaire qui peut être subclinique ou associée à des hémorragies cérébrales.

Combien de cas cela représente-t-il ? Si on additionne tous les cas décrits jusqu'à aujourd'hui (mars 2021), il s'agirait de 75 cas (Table 1). Ce chiffre est faible si on compare aux 470 cas de transmission iatrogène de maladie à prion découverts entre 1970 et aujourd'hui (30). Il est élevé, si on considère que la plupart des cas de transmission d'amyloïde ont été décrits à partir de 2015. Les mesures en place contre la transmission des maladies à prion lors d'actes chirurgicaux (7) limitent également fortement les risques de nouvelles contaminations par l'amyloïde- $\beta$. Comme les prions, l'amyloïde adhère fortement aux métaux et résiste aux procédures de stérilisation standard (31). Il convient donc d'utiliser au maximum des instruments à usage unique. Une autre mesure importante consiste à ne pas décontaminer les instruments de chirurgie avec des aldéhydes, des températures élevées ou d'autres agents qui tendent à fixer les souillures résiduelles sur les instruments. Le "bon sens" consisterait également à supprimer l'utilisation des mêmes instruments pour des actes impliquant des patients âgés ou adultes et ceux impliquant des enfants ou des jeunes adultes. Il conviendrait également de mettre en place des mesures de traçage des actes et instruments utilisés lors de chirurgies chez des patients qui ont évolué vers des maladie d'Alzheimer ou des syndromes apparentés (31).

\section{INTERET POUR LA RECHERCHE BIOMEDICALE : TRANSMISSION DE} PATHOLOGIE AMYLOÏDE

L'inoculation d'échantillons de cerveaux humains contenant des dépôts amyloïdes dans le cerveau de souris qui expriment de l'amyloïde accélère l'apparition de la pathologie amyloïde localement ou à distance du site d'injection chez la souris hôte (32). Ces travaux ont ouvert la possibilité de caractériser les mécanismes de transmission de la pathologie. Par exemple, ils montrent que les graines d'A $\beta$ injectées par voie périphérique (intraveineuse ou intrapéritonéale) peuvent induire une amylö̈dose parenchymale et surtout une angiopathie amyloïde cérébrale (33-35). Ce résultat est cohérent avec les suspicions de contamination par voie périphérique chez les patients ayant reçus de l'hormone de croissance par voie sous-cutanée (25) ou suite à des embolisations de tumeurs périphériques avec des extraits de dure-mère (36). 
196 Les études épidémiologiques chez l'homme ne permettent pas d'identifier précisément la nature

197 de l'amyloïde dans les éléments contaminants. Intuitivement, on peut penser que les plaques ou 198 l'angiopathie amyloïdes sont les contaminants principaux. Notre groupe vient de publier un article qui montre que l'inoculation d'échantillons de cerveaux en apparence sains peut également induire des lésions $\mathrm{A} \beta$. Pour arriver à cette conclusion, des homogénats de cerveaux humains riches en plaques amyloïdes ont été inoculés à un modèle murin résistant aux dépôts d'A $\beta$. Plus de 18 mois après l'inoculation, les animaux n'avaient pas développé de lésions amyloïdes dans certaines régions du cerveau qui ont ensuite été inoculées à un second modèle plus apte à développer des lésions $\mathrm{A} \beta$. Ce dernier a développé des plaques $\beta$-amyloïdes, ce qui suggère que des "graines" d'A $\beta$ peuvent persister sous des formes furtives dans les tissus cérébraux tout en conservant leur capacité à favoriser les dépôts d'A $\beta$ chez des hôtes réceptifs (37).

Les travaux chez des modèles rongeurs ont également mis en avant le concept de souches. Ce concept fait référence à la capacité de différents agents à induire des maladies avec des temps d'incubation distincts, des expressions cliniques et des lésions histopathologiques différentes. La transmission d'une souche d'agent pathogène dans un hôte différent conduit à son adaptation et peut parfois induire des phénotypes qui évoluent au cours de ce processus d'adaptation. Par exemple, l'adaptation de prions à des souris (38) ou à des macaques (39) peut induire des encéphalopathies en l'absence de lésions prion-positives. Dans le contexte de la maladie d'Alzheimer, il pourrait exister différentes "souches" de protéines amyloïdes (40). Ces souches pourraient induire des lésions différentes $(41,42)$ avec des conséquences

217 fonctionnelles différentes (42).

4. INTERET POUR LA RECHERCHE BIOMEDICALE : TRANSMISSION DE PATHOLOGIE TAU

Les modèles animaux les plus utilisés pour étudier la pathologie tau sont des souris transgéniques qui sur-expriment des protéines tau mutées. L'inoculation d'extraits de cerveaux humains à ces souris permet d'accélérer l'apparition d'une pathologie tau (43). De façon intéressante, en plus d'un processus de nucléation locale, la pathologie diffuse à distance du site d'injection (43). Ce processus rappelle la diffusion de la pathologie tau dans le cerveau humain au cours de la maladie d'Alzheimer (12) et renforce l'hypothèse de propagation des protéines tau le long des voies nerveuses. Un paradoxe apparent mérite d'être souligné : la pathologie tau est particulièrement transmissible chez les rongeurs alors que les études chez l'homme ne montrent pas de transmission. Nous ne pouvons aujourd'hui que spéculer sur l'origine de cette 
différence. Une hypothèse pourrait être que la transmission de tau est induite par des fractions

230 très insolubles du cerveau (44), qui peut-être ne sont pas présentes dans les contaminants 231 humains (extraits de dure-mère, hormone de croissance, ou outils chirurgicaux contaminés). 232 L'amyloïde est, elle, particulièrement transmise par des formes solubles d'A $\beta$ (45) qui 233 pourraient être plus présentes dans les contaminants.

234 Les mutations utilisées pour induire des pathologies tau chez les souris transgéniques 235 sont celles qui existent dans des démences fronto-temporales mais pas dans la maladie 236 d'Alzheimer. La pertinence de ces modèles pour étudier la maladie d'Alzheimer peut donc être 237 discutée. Des premiers travaux menés en 2013 ont révélé qu'il est possible d'induire une 238 pathologie tau chez des souris de type sauvage (qui ne surexpriment pas tau) en inoculant des cerveaux humains Alzheimer (43). Ces modèles seraient beaucoup plus pertinents que les modèles transgéniques actuels. La pathologie tau induite chez des souris de type sauvage peut être amplifiée en inoculant des extraits de cerveaux Alzheimer enrichis en tau (partie de cerveau insoluble dans le détergent ionique N-lauryl-sarcosine (sarkosyl)) (46, 47). Cette induction permet d'évaluer la pathologie tau dans un contexte d'expression physiologique des protéines et permettra de mieux comprendre les mécanismes physiopathologiques associés à la pathologie tau.

Comme pour l'amyloïde, le concept de souches est souligné pour les protéines tau. Par exemple, des extraits tau-positifs provenant de malades d'Alzheimer ou touchés par d'autres tauopathies (paralysie supra-nucléaire progressive, dégénérescence cortico-basale, maladie des grains argyrophiles ou maladie de Pick) induisent des lésions tau positives différentes qui rappellent les lésions de la pathologie des inoculas humains (43). PLUS INTEGATIFS DE LA MALADIE D'ALZEIMER

Une des difficultés dans la recherche sur la maladie d'Alzheimer est l'absence de modèles pertinents de l'interaction entre amyloïde et tau. La possibilité d'induire ces pathologies à partir d'inoculation de cerveaux humains est une étape importante pour comprendre la maladie.

256 Par exemple, la démonstration que l'inoculation d'extraits de cerveau Alzheimer enrichis en tau 257 (insolubles en sarkosyl) dans des modèles murins avec une production élevée d'A $\beta$ augmente 258 fortement la pathologie tau par rapport à des modèles sans $A \beta(48,49)$. Cela suggère que la 259 présence d'A $\beta$ crée un environnement qui amplifie les lésions tau. Ces nouveaux modèles permettent notamment d'évaluer des interactions entre $A \beta$ et tau au niveau 
microenvironnemental. Ils ont révélé que la réduction de la microglie au niveau des plaques amyloïdes favorise la nucléation et la propagation de tau (50). La transmission iatrogène de la pathologie amyloïde et tau permet également de créer des modèles primates qui développent un processus neurodégénératif plus marqué que les modèles rongeurs (29). Ces travaux permettront d'évaluer plus précisément les mécanismes associés au processus neurodégénératifs qui se produisent au cours de la maladie d'Alzheimer.

\section{ORIGINE DE LA TRANSMISSION IATROGENE : AU DELA DES MECANISMES} DE TYPE PRION

Les agents pathogènes à l'origine des maladies à prions sont constitués d'une protéine dont la conformation est anormale (51). Les protéines prions normales ont une conformation riche en hélices alpha. Elles peuvent être transformées en conformères insolubles, riches en hélices $\beta$ et ainsi devenir pathologiques et infectieuses. Ces changements conformationnels peuvent se produire de façon spontanée et on parle alors de nucléation primaire. Cette nucléation primaire est lente. Au cours des formes alimentaires/iatrogènes de maladies à prion, l'apport externe d'une protéine mal-conformée induit le changement de conformation de protéines endogènes normales pour les rendre pathologiques (processus de nucléation secondaire). Contrairement à la nucléation primaire, les processus de nucléations secondaires se produisent rapidement. Cela induit une augmentation rapide de la pathologie qui se répand dans le cerveau (52). L'hypothèse prédominante pour expliquer la transmission iatrogène ou expérimentale des protéines $\beta$-amyloïde et tau est que les protéines transmises se comportent "comme" des prions et que leur transmission est liée à l'induction de changements de conformation des protéines endogènes. Même si cette hypothèse est séduisante, d'autres possibilités sont envisageables. Par exemple, l'administration de protéines A $\beta$ pourrait déréguler/accélérer la synthèse d'A $\beta$ (53). L'augmentation de la concentration de l'A $\beta$ entrainerait alors une agrégation des protéines (54) et en parallèle l'initiation d'un cycle menant à plus de sécrétion d'amyloïde (55). Il est aussi possible que ce soit la toxicité de l'amyloïde sur les cellules qui entraine une augmentation de la synthèse de cet amyloïde.

\section{GENERALISATION AUX AUTRES MALADIES NEURODEGENERATIVES}

L'accumulation intracérébrale de protéines anormales intervient dans plusieurs autres maladies neurodégénératives et l'hypothèse de mécanismes de type prion (nucléation, propagation, transmissions) est également proposée pour expliquer la physiopathologie de ces maladies. L'alpha-synucléine est une protéine qui s'accumule dans le corps cellulaire des 
neurones et dans les neurites (lésions appelées corps et neurites de Lewy) au cours de la maladie

294 de Parkinson et de la démence à corps de Lewy (56). Au cours de l'atrophie multisystématisée, elle s'accumule dans les oligodendrocytes (57). Comme pour les lésions tau de la maladie d'Alzheimer, une progression chronologique des lésions alpha-synucléine-positives est décrite dans la maladie de Parkinson. Ces lésions progressent en touchant successivement le noyau dorsal du nerf vague et la zone réticulée intermédiaire dans le bulbe rachidien (stade 1) puis le complexe coeruleus-subcoeruleus dans le pont (stade 2), le mésencéphale dont la substance noire, les noyaux tubéromammillaires de l'hypothalamus et les noyaux magnocellulaires de la base du cerveau (stade 3), le néocortex temporal interne et en particulier le cortex entorhinal et l'allocortex (région CA2 de hippocampe) (stade 4), les néocortex associatifs pariétaux polymodaux et frontaux (stade 5) et les cortex prémoteurs, primaires ou associatifs unimodaux (stade 6) $(56,58)$. Ces lésions débutent de façon encore plus précoce dans le système nerveux entérique qui communique avec le nerf vague, ce qui a amené à l'hypothèse que la pathologie pourrait, dans certain cas, débuter dans les organes périphériques (59). Comme pour la pathologie amyloïde et tau, l'inoculation d'extraits de cerveaux humains touchés par la maladie de Parkinson permet d'induire l'accumulation intracellulaire d'alpha-synucléine pathologique chez des souris et des singes ainsi qu'un processus neurodégénératif (60), ce qui ouvre la voie à de nouveaux modèles de la maladie. La transmission d'alpha-synucléine semble être encore plus efficace à partir d'extraits de cerveaux de patients atteints d'atrophie multisystématisée (61). La transmission iatrogène d'alpha-synucléine n'a cependant pas été mise en évidence chez l'homme. Des cas exceptionnels de transmission d'alpha-synucléine de cerveaux de patients parkinsoniens à des neurones fétaux greffés à ces patients (plus de 10 ans après la greffe) suggèrent une possible transmission d'alpha-synucléine (62).

La TDP-43 (TAR DNA-binding 43) est une autre protéine associée aux maladies neurodégénératives (certaines formes de démence fronto-temporale, démence sémantique). Sa transmission a également été mise en évidence chez des modèles rongeurs après inoculation d'extraits de cerveaux humains TDP-43 positifs (63), étendant encore plus le spectre des protéines transmissibles. Comme pour l'alpha-synucléine, la transmission iatrogène a l'homme n'a cependant pas été rapportée.

\section{CONCLUSION}

De nombreux éléments épidémiologiques et expérimentaux démontrent que des lésions amyloïdes peuvent être transmises de façon iatrogène chez l'homme. Ces lésions n'induisent pas un tableau clinique de maladie d'Alzheimer, mais plutôt une angiopathie amyloïde avec 
hémorragies cérébrales chez des personnes jeunes. En présence de ce tableau clinique, une enquête doit être menée pour tracer les chirurgies ou autres facteurs de risques du patient. Des études de cohortes d'individus à risque, par exemple ceux ayant bénéficié d'une chirurgie cérébrale en étant jeunes, sont aussi en cours de construction. Elles devront être suivies par imagerie et examens cognitifs afin de caractériser les conséquences d'une potentielle contamination. Aujourd'hui les caractéristiques des tissus contaminants restent spéculatives. Par exemple, il est possible que des tissus contaminants présentent peu de plaques amyloïdes. Au-delà du problème clinique, cette transmission permet d'explorer des nouveaux paradigmes explicatifs de la maladie d'Alzheimer et de développer des nouveaux modèles animaux, plus valides que les modèles transgéniques actuels. Leur utilisation permettra de mieux comprendre la maladie et de développer des nouvelles thérapies contre la maladie d'Alzheimer.

\section{CONFLITS D'INTERET}

L'auteur déclare ne pas avoir de liens d'intérêts.

\section{RÉFÉRENCES}

340 1. Gajdusek DC, Gibbs CJ, Alpers M. Experimental transmission of a Kuru-like syndrome 341 to chimpanzees. Nature. 1966 Feb 19;209(5025):794-6. PMID: 5922150.

342 2. Alpers MP. The epidemiology of kuru in the period 1987 to 1995 Comm Dis Int. 343 2005;29:391-9.

344 3. Uttley L, Carroll C, Wong R, Hilton DA, Stevenson M. Creutzfeldt-Jakob disease: a systematic review of global incidence, prevalence, infectivity, and incubation. Lancet Infect Dis. 2020 Jan;20(1):E2-E10. PMID: WOS:000504005300004.

4. Brown P, Brandel J-P, Sato T, Nakamura Y, MacKenzie J, Will RG, et al. Iatrogenic Creutzfeldt-Jakob disease, final assessment. Emerg Infect Dis. 2012 Jun;18(6):901-7. PMID: 22607808.

5. Brooke FJ, Boyd A, Klug GM, Masters CL, Collins SJ. Lyodura use and the risk of iatrogenic Creutzfeldt-Jakob disease in Australia. Med J Australia. 2004 Feb 16;180(4):177-81. PMID: WOS:000189133500009.

6. Antoine JC, Michel D, Bertholon P, Mosnier JF, Laplanche JL, Beaudry P, et al. Creutzfeldt-Jakob disease after extracranial dura mater embolization for a nasopharyngeal angiofibroma. Neurology. 1997 May;48(5):1451-3. PMID: WOS:A1997WZ77800057.

7. Ministère du Travail, de l'Emploi et de la santé; Ministère des solidarité et de la cohésion sociale. Instruction DGS/R13 no 2011-449 du 1er décembre 2011 relative à 1'actualisation des recommandations visant à réduire les risques de transmission d'agents transmissibles non conventionnels lors des actes invasifs. Bulletin Officiel Santé - Protection sociale - Solidarité. 2012; 2012/1 - 15 février 2012.

8. Bateman RJ, Xiong C, Benzinger TL, Fagan AM, Goate A, Fox NC, et al. Clinical and biomarker changes in dominantly inherited Alzheimer's disease. N Engl J Med. 2012 Aug 30;367(9):795-804. PMID: 22784036.

9. Duyckaerts C, Delatour B, Potier MC. Classification and basic pathology of Alzheimer disease. Acta Neuropathol. 2009 Jul;118(1):5-36. PMID: 19381658. 
10. Cleary JP, Walsh DM, Hofmeister JJ, Shankar GM, Kuskowski MA, Selkoe DJ, et al. Natural oligomers of the amyloid-beta protein specifically disrupt cognitive function. Nat Neurosci. 2005 Jan;8(1):79-84. PMID: 15608634. Jackson GR, et al. Identification of oligomers at early stages of tau aggregation in Alzheimer's disease. Faseb J. 2012 May;26(5):1946-59. PMID: 22253473.

12. Braak H, Braak E. Neuropathological stageing of Alzheimer related changes. Acta Neuropathol. 1991;82:239-59.

13. Delacourte A, David JP, Sergeant N, Buee L, Wattez A, Vermersch P, et al. The biochemical pathway of neurofibrillary degeneration in aging and Alzheimer's disease. Neurology. 1999;52(6):1158-65.

14. Thal DR, Rub U, Orantes M, Braak H. Phases of A beta-deposition in the human brain and its relevance for the development of AD. Neurology. 2002 Jun 25;58(12):1791-800. PMID: 12084879.

15. Beekes M, Thomzig A, Schulz-Schaeffer WJ, Burger R. Is there a risk of prion-like disease transmission by Alzheimer- or Parkinson-associated protein particles? Acta Neuropathol. 2014 Oct;128(4):463-76. PMID: 25073522.

16. Edgren G, Hjalgrim H, Rostgaard K, Lambert P, Wikman A, Norda R, et al. Transmission of Neurodegenerative Disorders Through Blood Transfusion: A Cohort Study. Ann Intern Med. 2016 Sep 06;165(5):316-24. PMID: 27368068.

17. Schmidt C, Karch A, Korth C, Zerr I. On the issue of transmissibility of Alzheimer disease: a critical review. Prion. 2012 Nov-Dec;6(5):447-52. PMID: 23052009.

18. Irwin DJ, Abrams JY, Schonberger LB, Leschek EW, Mills JL, Lee VM, et al. Evaluation of potential infectivity of Alzheimer and Parkinson disease proteins in recipients of cadaver-derived human growth hormone. JAMA neurology. 2013 Apr;70(4):462-8. PMID: 23380910.

19. Preusser M, Strobel T, Gelpi E, Eiler M, Broessner G, Schmutzhard E, et al. Alzheimertype neuropathology in a 28 year old patient with iatrogenic Creutzfeldt-Jakob disease after dural grafting. J Neurol Neurosur Ps. 2006 Mar;77(3):413-6. PMID: WOS:000235651900034. 20. Jaunmuktane Z, Mead S, Ellis M, Wadsworth JD, Nicoll AJ, Kenny J, et al. Evidence for human transmission of amyloid-beta pathology and cerebral amyloid angiopathy. Nature. 2015 Sep 10;525(7568):247-50. PMID: 26354483.

21. Duyckaerts C, Sazdovitch V, Ando K, Seilhean D, Privat N, Yilmaz Z, et al. Neuropathology of iatrogenic Creutzfeldt-Jakob disease and immunoassay of French cadaversourced growth hormone batches suggest possible transmission of tauopathy and long incubation periods for the transmission of Abeta pathology. Acta Neuropathol. 2018 Feb;135(2):201-12. PMID: 29209767.

22. Purro SA, Farrow MA, Linehan J, Nazari T, Thomas DX, Chen ZC, et al. Transmission of amyloid-beta protein pathology from cadaveric pituitary growth hormone. Nature. 2018 Dec 20;564(7736):415-+. PMID: WOS:000453834900061.

23. Frontzek K, Lutz MI, Aguzzi A, Kovacs GG, Budka H. Amyloid-beta pathology and cerebral amyloid angiopathy are frequent in iatrogenic Creutzfeldt-Jakob disease after dural grafting. Swiss medical weekly. 2016;146:w14287. PMID: 26812492.

24. Kovacs GG, Lutz MI, Ricken G, Strobel T, Hoftberger R, Preusser M, et al. Dura mater is a potential source of Abeta seeds. Acta Neuropathol. 2016 Mar 25;131(6):911-23. PMID: 27016065.

25. Ritchie DL, Adlard P, Peden AH, Lowrie S, Le Grice M, Burns K, et al. Amyloid-beta accumulation in the CNS in human growth hormone recipients in the UK. Acta Neuropathol. 2017 Aug;134(2):221-40. PMID: WOS:000405315500005. 

Abeta cerebral amyloid angiopathy 4 decades after a dural graft at the age of 2 years. Acta Neuropathol. 2018 Mar 5;135(5):801-3. PMID: 29508058.

418 27. Jaunmuktane Z, Quaegebeur A, Taipa R, Viana-Baptista M, Barbosa R, Koriath C, et al. Evidence of amyloid-beta cerebral amyloid angiopathy transmission through neurosurgery. Acta Neuropathol. 2018 May;135(5):671-9. PMID: 29450646.

28. Ridley RM, Baker HF, Windle CP, Cummings RM. Very long term studies of the seeding of beta-amyloidosis in primates. J Neural Transm. 2006 Sep;113(9):1243-51. PMID: 16362635.

29. Gary C, Lam S, Herard AS, Koch JE, Petit F, Gipchtein P, et al. Encephalopathy induced by Alzheimer brain inoculation in a non-human primate. Acta Neuropathol Commun. 2019;7(126).

30. Duffy P, Wolf J, Collins G, DeVoe AG, Streeten B, Cowen D. Letter: Possible personto-person transmission of Creutzfeldt-Jakob disease. N Engl J Med. 1974 Mar 21;290(12):6923. PMID: 4591849.

31. Lauwers E, Lalli G, Brandner S, Collinge J, Compernolle V, Duyckaerts C, et al. Potential human transmission of amyloid beta pathology: surveillance and risks. Lancet Neurol. 2020 Oct;19(10):872-8. PMID: WOS:000581119100024.

32. Jucker M, Walker LC. Pathogenic protein seeding in Alzheimer disease and other neurodegenerative disorders. Ann Neurol. 2011 Oct;70(4):532-40. PMID: 22028219.

33. Eisele YS, Bolmont T, Heikenwalder M, Langer F, Jacobson LH, Yan ZX, et al. Induction of cerebral beta-amyloidosis: intracerebral versus systemic Abeta inoculation. Proc Natl Acad Sci USA. 2009 Aug 4;106(31):12926-31. PMID: 19622727.

34. Eisele YS, Fritschi SK, Hamaguchi T, Obermuller U, Fuger P, Skodras A, et al. Multiple factors contribute to the peripheral induction of cerebral beta-amyloidosis. J Neurosci. 2014 Jul 30;34(31):10264-73. PMID: 25080588.

35. Burwinkel M, Lutzenberger M, Heppner FL, Schulz-Schaeffer W, Baier M. Intravenous injection of beta-amyloid seeds promotes cerebral amyloid angiopathy (CAA). Acta Neuropathol Commun. 2018 Mar 5;6. PMID: WOS:000426661800001.

36. Banerjee G, Adams ME, Jaunmuktane Z, Lammie GA, Turner B, Wani M, et al. Early onset cerebral amyloid angiopathy following childhood exposure to cadaveric dura. Ann Neurol. 2019 Feb;85(2):284-90. PMID: WOS:000457448500013.

37. Herard AS, Petit F, Gary C, Guillermier M, Boluda S, Garin CM, et al. Induction of amyloid-beta deposits from serially transmitted, histologically silent, A-beta seeds issued from human brains. Acta Neuropathol Commun. 2020;8:Article number: 205.

38. Lasmezas CI, Deslys JP, Robain O, Jaegly A, Beringue V, Peyrin JM, et al. Transmission of the BSE agent to mice in the absence of detectable abnormal prion protein. Science. 1997 Jan 17;275(5298):402-5. PMID: 8994041.

39. Comoy EE, Mikol J, Jaffre N, Lebon V, Levavasseur E, Streichenberger N, et al. Experimental transfusion of variant CJD-infected blood reveals previously uncharacterised prion disorder in mice and macaque. Nat Commun. 2017 Nov 2;8. PMID: WOS:000414245200008.

40. Meyer-Luehmann M, Coomaraswamy J, Bolmont T, Kaeser S, Schaefer C, Kilger E, et al. Exogenous induction of cerebral beta-amyloidogenesis is governed by agent and host. Science. 2006 Sep 22;313(5794):1781-4. PMID: 16990547.

41. Watts JC, Condello C, Stohr J, Oehler A, Lee J, DeArmond SJ, et al. Serial propagation of distinct strains of Abeta prions from Alzheimer's disease patients. Proc Natl Acad Sci USA. 2014 Jul 15;111(28):10323-8. PMID: 24982139. 

subtypes of Alzheimer's disease. Scientific Reports. 2018 Feb 19;8. PMID: WOS:000425380900052. Artn 3269. 43. Clavaguera F, Akatsu H, Fraser G, Crowther RA, Frank S, Hench J, et al. Brain homogenates from human tauopathies induce tau inclusions in mouse brain. Proc Natl Acad Sci USA. 2013 Jun 4;110(23):9535-40. PMID: 23690619. Transmission and spreading of tauopathy in transgenic mouse brain. Nat Cell Biol. 2009 Jul;11(7):909-13. PMID: 19503072.

45. Langer F, Eisele YS, Fritschi SK, Staufenbiel M, Walker LC, Jucker M. Soluble Abeta seeds are potent inducers of cerebral beta-amyloid deposition. J Neurosci. 2011 Oct 12;31(41):14488-95. PMID: 21994365.

46. Audouard E, Houben S, Masaracchia C, Yilmaz Z, Suain V, Authelet M, et al. HighMolecular-Weight Paired Helical Filaments from Alzheimer Brain Induces Seeding of WildType Mouse Tau into an Argyrophilic 4R Tau Pathology in Vivo. Am J Pathol. 2016 Aug 3. PMID: 27497324.

47. Guo JL, Narasimhan S, Changolkar L, He ZH, Stieber A, Zhang B, et al. Unique pathological tau conformers from Alzheimer's brains transmit tau pathology in nontransgenic mice. J Exp Med. 2016 Nov;213(12):2635-54. PMID: WOS:000391122400009.

48. He ZH, Guo JL, McBride JD, Narasimhan S, Kim H, Changolkar L, et al. Amyloid-beta plaques enhance Alzheimer's brain tau-seeded pathologies by facilitating neuritic plaque tau aggregation. Nature Medicine. 2018 Jan;24(1):29-+. PMID: WOS:000422965900008.

49. Vergara C, Houben S, Suain V, Yilmaz Z, De Decker R, Vanden Dries V, et al. Amyloid- pathology enhances pathological fibrillary tau seeding induced by Alzheimer PHF in vivo. Acta Neuropathol. 2019 Mar;137(3):397-412. PMID: WOS:000460009000003.

50. Leyns CEG, Gratuze M, Narasimhan S, Jain N, Koscal LJ, Jiang H, et al. TREM2 function impedes tau seeding in neuritic plaques. Nat Neurosci. 2019 Aug;22(8):1217-+. PMID: WOS:000477065500005.

51. Prusiner SB. Novel proteinaceous infectious particles cause scrapie. Science. 1982 Apr 9;216(4542):136-44. PMID: 6801762.

52. Eisele YS. From soluble abeta to progressive abeta aggregation: could prion-like templated misfolding play a role? Brain Pathol. 2013 May;23(3):333-41. PMID: 23587139.

53. Pietri M, Dakowski C, Hannaoui S, Alleaume-Butaux A, Hernandez-Rapp J, Ragagnin A, et al. PDK1 decreases TACE-mediated alpha-secretase activity and promotes disease progression in prion and Alzheimer's diseases. Nature Medicine. 2013 Sep;19(9):1124-31. PMID: WOS:000324083500026.

54. Burgold S, Filser S, Dorostkar MM, Schmidt B, Herms J. In vivo imaging reveals sigmoidal growth kinetic of beta-amyloid plaques. Acta Neuropathol Commun. 2014;2:30. PMID: 24678659.

55. Rolland M, Powell R, Jacquier-Sarlin M, Boisseau S, Reynaud-Dulaurier R, MartinezHernandez $J$, et al. Interaction of A $\beta$ oligomers with neuronal APP triggers a vicious cycle leading to the propagation of synaptic plasticity alterations to healthy neurons. J Neurosci. 2020;40(27):5161-76. PMID: 32444385.

56. Duyckaerts C, Sazdovitch V, Seilhean D. Évolution des connaissances sur le processus pathologique de la maladie de Parkinson. Bulletin de l'Academie nationale de medecine. 2010;194(7):1287-304.

57. Burn DJ, Jaros E. Multiple system atrophy: cellular and molecular pathology. J Clin Pathol-Mol Pa. 2001 Dec;54(6):419-26. PMID: WOS:000172762500010. 
511 58. Braak H, Del Tredici K, Rub U, de Vos RAI, Steur ENHJ, Braak E. Staging of brain 512 pathology related to sporadic Parkinson's disease. Neurobiol Aging. 2003 Mar-Apr;24(2):197513 211. PMID: WOS:000180616100001.

514 59. Travagli RA, Browning KN, Camilleri M. Parkinson disease and the gut: new insights 515 into pathogenesis and clinical relevance. Nat Rev Gastro Hepat. 2020 Nov;17(11):673-85. 516 PMID: WOS:000559358800001.

517 60. Recasens A, Dehay B, Bove J, Carballo-Carbajal I, Dovero S, Perez-Villalba A, et al. 518 Lewy body extracts from Parkinson disease brains trigger alpha-synuclein pathology and 519 neurodegeneration in mice and monkeys. Ann Neurol. 2014 Mar;75(3):351-62. PMID: 520 WOS:000333547000006.

521 61. Prusiner SB, Woerman AL, Mordes DA, Watts JC, Rampersaud R, Berry DB, et al. 522 Evidence for alpha-synuclein prions causing multiple system atrophy in humans with 523 parkinsonism. Proc Natl Acad Sci USA. 2015 Aug 31. PMID: 26324905.

524 62. Li JY, Englund E, Holton JL, Soulet D, Hagell P, Lees AJ, et al. Lewy bodies in grafted 525 neurons in subjects with Parkinson's disease suggest host-to-graft disease propagation. Nat Med. 5262008 May;14(5):501-3. PMID: 18391963.

527 63. Porta S, Xu Y, Restrepo CR, Kwong LK, Zhang B, Brown HJ, et al. Patient-derived 528 frontotemporal lobar degeneration brain extracts induce formation and spreading of TDP-43 529 pathology in vivo. Nat Commun. 2018 Oct 11;9. PMID: WOS:000447075700008. 
TABLE

\begin{tabular}{|c|c|c|c|c|c|c|c|}
\hline & \multicolumn{4}{|c|}{ Procédures chirurgicales } & \multicolumn{3}{|c|}{ Procédures médicales } \\
\hline & Greffe de dure-mère & $\begin{array}{c}\text { Chirurgie cérébrale } \\
\text { avec presomption de } \\
\text { contamination via les } \\
\text { instruments }\end{array}$ & $\begin{array}{c}\text { Electro- } \\
\text { encephalogramme } \\
\text { (contamination par } \\
\text { aiguille d'EEG) }\end{array}$ & $\begin{array}{l}\text { Transplantation } \\
\text { cornéenne }\end{array}$ & $\begin{array}{l}\text { Hormone de } \\
\text { croissance }\end{array}$ & $\begin{array}{c}\text { Hormone } \\
\text { gonadotrophique }\end{array}$ & $\begin{array}{c}\text { Produits dérivés du } \\
\text { sang }\end{array}$ \\
\hline Prion : 470 cas & 228 & 4 & 2 & 2 & 226 & 4 & 3 \\
\hline \multirow[t]{2}{*}{$\begin{array}{l}\text { Transmission d'amyloìde- } \\
\beta \\
75 \text { cas }\end{array}$} & $\begin{array}{c}\text { Preusser, } 2006[1] \\
\text { Frontzek, } 2016[5] \\
\text { Kovacs, } 2016[2] \\
\text { Hamaguchi, 2016 [11] } \\
\text { Iwasaki [1] } \\
\text { Herve, } 2018[1] \\
\text { Cali, } 2018[8] \\
\text { Banerjee, } 2019[3] \\
\text { Raposo, } 2020[1] \\
\end{array}$ & $\begin{array}{c}\text { Jaunmuktane, } 2018[8] \\
\text { Hamaguchi, } 2019[2] \\
\text { Giaccone, } 2019[1]\end{array}$ & & & $\begin{array}{c}\text { Jaunmuktane, } 2015[4] \\
\text { Duyckaerts, } 2018[1] \\
\text { Ritchie, } 2017[23] \\
\text { Cali, } 2018[3]\end{array}$ & & \\
\hline & $\begin{array}{c}\text { Total : } 33 \\
\text { (dont } 5 \text { cas avec } \\
\text { hémorragies) }\end{array}$ & $\begin{array}{c}\text { Total : } 11 \\
\text { (dont } 11 \text { cas avec } \\
\text { hémorragies) }\end{array}$ & Total : 0 & Total : 0 & Total : 31 & Total : 0 & Total : 0 \\
\hline
\end{tabular}

Table 1 : Comparaison des procédures médico-chirurgicales ayant conduit à l'induction iatrogène de maladies à prions et d'amylö̈de- $\beta$. Les articles citant l'induction d'amyloïde sont présentés, et le nombre de cas d'induction est précisé entre crochets. Les articles notés en rouge rapportent des hémorragies cérébrales avec conséquences cliniques. 